\title{
REVISIÓN METODOLÓGICA DEL CÁLCULO DE LA LETRA L DEL PRODUCTO BRUTO GEOGRÁFICO DE CHACO SERIE 2004-2015
}

\section{METHODOLOGICAL REVIEW OF LETTER L CALCULATION IN GROSS GEOGRAPHIC PRODUCT OF CHACO SERIES 2004-2015}

\author{
Alcira Patricia Soria Ojeda \\ Facultad de Ciencias Económicas \\ Universidad Nacional del Nordeste \\ Resistencia, Chaco, Argentina \\ alcira.soria.ojeda@comunidad.unne.edu.ar
}

\author{
Julieta Bermúdez \\ Facultad de Ciencias Económicas \\ Universidad Nacional del Nordeste \\ Resistencia, Chaco, Argentina \\ juulibermudez@gmail.com
}

Fecha de recepción: 10/09/2020 - Fecha de revisión: 29/12/2020 - Fecha de aprobación: 04/01/2021

DOI: https://doi.org/10.36995/.visiondefuturo.2021.25.02.004.es

\section{RESUMEN}

En 2013, se realizó el cambio de año base del Producto Bruto Geográfico (PBG) del Chaco, efectuándose antes del cambio de base del Producto Interno Bruto; por lo que la modificación provincial se realizó sin contar con la metodología propuesta luego por el Instituto Nacional de Estadística y Censos (INDEC) para el cálculo del indicador nacional.

Así, esta ponencia está enfocada en la definición de ajustes metodológicos para mejorar el cálculo de la letra $L$ (Administración pública y defensa, y planes de seguridad social de afiliación obligatoria) del PBG provincial.

Se presenta el análisis del cálculo actual, comparando con la metodología propuesta por la Dirección Nacional de Cuentas Nacionales del INDEC y las definiciones de organismos internacionales para la estimación de este sector de actividad. Asimismo, se realiza una comparación del clasificador usado actualmente con la última versión del sugerido por Naciones Unidas.

Se concluye que ambas metodologías no presentan significativas discrepancias, pero se realizan recomendaciones para mejorar las estimaciones del nivel municipal, de la rama de seguridad social y del gasto de la administración nacional correspondiente al Chaco; y para enriquecer el uso de índices. A su vez, la comparación de los clasificadores no evidencia cambios significativos, pero justifica la necesidad de actualización.

PALABRAS CLAVE: Producto Bruto Geográfico; Administración Pública; Metodología de Cálculo; Clasificador de Actividades.

\section{ABSTRACT}

In 2013, the base year change of the Chaco's Gross Geographical Product was made, taking place before the base change of the Gross Domestic Product. Therefore, the provincial

\footnotetext{
"Visión de Futuro" Año 18, Volumen No 25 № 2, Edición Especial-III Congreso Regional de Economía del Norte Grande-Pág 80-100 URL de la Revista: $\underline{\text { http://visiondefuturo.fce.unam.edu.ar/index.php/visiondefuturo/index }}$

URL del Documento: https://visiondefuturo.fce.unam.edu.ar/index.php/visiondefuturo/issue/view/20 
modification was carried out without having the methodology proposed later by the National Institute of Statistics and Censuses (INDEC) for the calculation of the national indicator.

Consequently, this presentation is focused on the definition of methodological adjustments to improve the calculation of the letter $L$ (Public administration and defense, and compulsory affiliation social security plans) of the Gross Geographical Product.

The analysis of the current calculation is presented, comparing it with the methodology proposed by INDEC and the definitions of international organizations for the estimation of this sector of activity. Likewise, a comparison is made of the classifier currently used with the latest version of the one suggested by the United Nations.

It is concluded that both methodologies do not present significant discrepancies, but recommendations are made to improve the estimates of the municipal level, the social security branch and the expenditure of the national administration corresponding to the Chaco; and to enrich the use of indexes. In turn, the comparison of the classifiers does not show significant changes, but justifies the need for updating.

KEY WORDS: Gross Geographical Product; Public Administration; Calculation Methodology; Activity Classifier.

\section{INTRODUCCIÓN}

Este documento forma parte de un proyecto de investigación de la Facultad de Ciencias Económicas de la Universidad Nacional del Nordeste (UNNE), aprobado por la Secretaría de Ciencia y Técnica de la UNNE bajo la denominación 17M002 "Hacia la construcción de estadísticas regionales actualizadas: Revisión y asignación geográfica del Producto Bruto Geográfico de Chaco".

Con tal proyecto se busca contribuir a la mejora de la metodología de cálculo que se utiliza en la provincia del Chaco para el Producto Bruto Geográfico (PBG), tomando como referencia la metodología utilizada a nivel nacional, así como las definiciones de organismos internacionales. También se pretende generar una asignación geográfica de dicho PBG dentro de la provincia de modo de establecer el valor generado por las distintas regiones. Para ello, se buscará determinar ciertos indicadores que permitan determinar esta distribución.

Por cuanto, el proyecto cuenta con los siguientes objetivos:

- Objetivo General:

- Generar una metodología local, ajustada a lineamientos establecidos por organismos nacionales e internacionales, para el cálculo de datos macroeconómicos confiables y actualizados, que permitan brindar información estadística que apoye la evaluación de alternativas, toma de decisiones y

\footnotetext{
“Visión de Futuro" Año 18, Volumen No 25 № 2, Edición Especial-III Congreso Regional de Economía del Norte Grande-Pág 80-100 URL de la Revista: http://visiondefuturo.fce.unam.edu.ar/index.php/visiondefuturo/index

URL del Documento: https://visiondefuturo.fce.unam.edu.ar/index.php/visiondefuturo/issue/view/20

ISSN 1668 - 8708 - Versión en Línea

E-mail: revistacientifica@fce.unam.edu.ar
} 
evaluación de resultados, en distintos niveles institucionales tanto públicos como privados.

- Objetivos Específicos:

- Determinar un criterio de asignación geográfica del valor agregado generado en la Provincia de Chaco.

- Contribuir al mejoramiento del cálculo del Producto Bruto Geográfico de la Provincia de Chaco.

- Desarrollar un equipo de expertos con competencias para asesorar, apoyar y contribuir con el buen funcionamiento del sistema estadístico provincial.

- Fomentar vínculos interinstitucionales que permiten a la universidad insertarse en espacios del medio que requieren su participación al tiempo que garanticen la confiabilidad de variables muy sensibles para la toma de decisiones que comprometen asignación de recursos.

Este proyecto cuenta con un periodo de ejecución de cuatro años (2018-2021). Este documento se encuadra en la primera etapa de la investigación que consiste en la comparación de metodologías de cálculo de cada una de las letras que integran el PBG de la provincia.

Asimismo, cabe mencionar que se ha trabajado en constante intercambio con la Dirección de Estadísticas y Censos de la Provincia de Chaco, institución que ha tenido a su cargo, históricamente, la elaboración de este indicador productivo, con el apoyo financiero del Consejo Federal de Inversiones de Argentina (CFI), por lo que esta interacción le permite al equipo contar con antecedentes relacionados con el cálculo del PBG.

Entonces, bajo estos lineamientos, este documento se enfoca específicamente en la comparación de las metodologías de cálculo de la Letra L, Administración pública, defensa y planes de seguridad social de afiliación obligatoria; utilizadas para las estimaciones del PBG de la Provincia del Chaco y del PIB de Argentina. Teniendo como objetivo contribuir al mejoramiento del cálculo de la letra L, en la estimación PBG provincial.

Se presenta en primer lugar una revisión de los principales conceptos relativos a las cuentas naciones y regionales. Luego, se describe el sector en cuestión detallando qué tipo de actividades son consideradas dentro de la Letra $L$.

Así, se llega al apartado correspondiente a la revisión metodológica, el cual cuenta con tres partes. La primera se refiere a la metodología de cálculo de la Letra $L$ del PBG del Chaco, y la segunda se refiere a la metodología del PIB. Ambas tratan los mismos aspectos: año base, estimación a precios constantes, estimación a precios corrientes, valuación y economía

\footnotetext{
"Visión de Futuro" Año 18, Volumen N²5 N² 2, Edición Especial-III Congreso Regional de Economía del Norte Grande-Pág 80-100 URL de la Revista: http://visiondefuturo.fce.unam.edu.ar/index.php/visiondefuturo/index

URL del Documento: https://visiondefuturo.fce.unam.edu.ar/index.php/visiondefuturo/issue/view/20

ISSN 1668 - 8708 - Versión en Línea

E-mail: revistacientifica@fce.unam.edu.ar
} 
no observada. Luego, la tercera parte hace referencia al análisis de los nomencladores de actividades.

Seguido a lo anterior, se exhibe un análisis de los datos de las series calculadas de la letra L para el PBG y el PIB para el periodo 2004-2015.

\section{DESARROLLO}

\section{Marco Conceptual. La Contabilidad Nacional y el Sistema de Cuentas Regionales}

Como se ha mencionado anteriormente, el objetivo de este trabajo es contribuir al mejoramiento del cálculo de la letra L: Administración pública y defensa, planes de seguridad social obligatoria; en la estimación del Producto Bruto Geográfico (PBG) de la Provincia de Chaco, realizando una revisión metodológica del cálculo del mismo, y comparando con la metodología utilizada por el Instituto Nacional de Estadísticas y Censos (INDEC) para el cálculo del Producto Interno Bruto (PIB).

Con el fin de comprender mejor el análisis que sigue a continuación, resulta relevante realizar una breve presentación de los conceptos más importantes referidos a un Sistema de Cuentas Regionales y a la estimación del producto para una región o provincia, en este caso.

EI PBG forma parte de lo que se denomina un Sistema de Cuentas Regionales. Para definir tal sistema, debemos recurrir en primer lugar a la definición de una Sistema de Cuentas Nacionales (SCN) y, previamente, al concepto y finalidad de la Contabilidad Nacional.

Siguiendo a Laveglia (2006), la Contabilidad Nacional, Social o Económica, es un registro sistemático de los hechos económicos que realizan las entidades de una unidad geopolítica en un período cronológico determinado.

Como explica Seruzier (2003), el propósito de la contabilidad nacional es responder a una necesidad experimentada por todos: informar de manera sintética sobre la situación económica de un país. Así, explica el autor que medir tal situación es una tarea difícil, dado que dentro de una economía se realizan diversas actividades y participan numerosos actores.

Pasando al concepto de SCN, Laveglia (2006) lo define como un conjunto coherente sistemático e integrado de cuentas macroeconómicas, balances y tablas basados en conceptos, definiciones, clasificaciones y reglas contables aceptadas internacionalmente. Siguiendo al autor, el sistema registra las actividades que tienen lugar en una economía y la interacción entre los agentes económicos; teniendo como objetivo "proporcionar un marco conceptual y contable completo que pueda utilizarse para crear una base de datos macroeconómica adecuada para el análisis y la evaluación de los resultados de una economía" (Laveglia, 2006, p. 13).

\footnotetext{
"Visión de Futuro" Año 18, Volumen No 25 º 2, Edición Especial-III Congreso Regional de Economía del Norte Grande-Pág 80-100 URL de la Revista: http://visiondefuturo.fce.unam.edu.ar/index.php/visiondefuturo/index

URL del Documento: https://visiondefuturo.fce.unam.edu.ar/index.php/visiondefuturo/issue/view/20

ISSN 1668 - 8708 - Versión en Línea

E-mail: revistacientifica@fce.unam.edu.ar
} 
Así, siguiendo a la Dirección Provincial de Estadística (2018) de la Provincia de Buenos Aires, uno de los principales indicadores utilizados para evaluar el funcionamiento de una economía es el Producto Bruto, es decir, el valor monetario de todos los bienes y servicios finales producidos por una economía durante un periodo de tiempo, generalmente un año. "El Producto Bruto recibe la denominación de Interno (PBI) cuando es generado por factores productivos residentes dentro de las fronteras de un país" (Dirección Provincial de Estadística, 2018, p. 11).

Teniendo en cuenta lo mencionado hasta aquí, el Sistema de Cuentas Regionales "se encuentra integrado al Sistema de Cuentas Nacionales aceptando las definiciones y normas establecidas por éste" (Laveglia, 2006, p. 36)

En este marco, cuando el producto bruto "está referido a una jurisdicción política subnacional (región, provincia o municipio) en Argentina, recibe el nombre de Producto Bruto Geográfico (PBG)" (Dirección Provincial de Estadística, 2018, p.11).

La Dirección de Estadísticas de la Provincia de Buenos Aires define al PBG, señalando que es el valor de los bienes y servicios finales producidos en una provincia, reflejando la actividad económica de las unidades productivas residentes en el territorio provincial.

\section{Descripción del Sector: Administración pública y defensa; y planes de seguridad social afiliación obligatoria}

Previo al tratamiento de las cuestiones relacionadas al cálculo de la Letra $L$ referida al sector correspondiente a la Administración pública y defensa, resulta necesario definir qué actividades quedan comprendidas dentro de este sector.

En este sentido es útil recurrir a la Clasificación Internacional Industrial Uniforme (CIIU) de todas las actividades económicas. Este clasificador es elaborado por las Naciones Unidas desde 1948 y es actualizado periódicamente, por lo que existen varias versiones del mismo. En el caso de Argentina, para el cálculo del PIB el país basa su propia clasificación de actividades (Clasificación Nacional de Actividades Económica 2004, ClaNAE 04), en el CIIU revisión 3.1.1

De acuerdo Naciones Unidas (2005), siguiendo al CIIU 3.1, la Letra L comprende las actividades que suelen correr a cargo de la administración pública, entre las que se cuentan la promulgación de leyes, su interpretación judicial y la administración de los reglamentos de aplicación y programas correspondientes, las actividades legislativas, tributarias, de defensa nacional y de orden público y seguridad, los servicios de inmigración, las relaciones exteriores

\footnotetext{
${ }^{1}$ Sobre el análisis de los diferentes clasificadores se volverá en el anteúltimo apartado.

"Visión de Futuro" Año 18, Volumen $N^{\circ} 25$ № 2, Edición Especial-III Congreso Regional de Economía del Norte Grande-Pág 80-100 URL de la Revista: http://visiondefuturo.fce.unam.edu.ar/index.php/visiondefuturo/index

URL del Documento: https://visiondefuturo.fce.unam.edu.ar/index.php/visiondefuturo/issue/view/20

ISSN 1668 - 8708 - Versión en Línea

E-mail: revistacientifica@fce.unam.edu.ar
} 
y la administración de programas gubernamentales; incluyendo las actividades de los planes de seguridad social de afiliación obligatoria."

Merece mención, tal como se detalla en Naciones Unidas (2009), la propia naturaleza de la actividad será el factor determinante para clasificarla dentro de esta sección, y no lo será la condición jurídica o institucional de las unidades que la realizan. "Eso significa que las actividades clasificadas en otras partes de la CIIU no se incluyen en esta sección, aunque las realicen entidades públicas." (Naciones Unidas, 2009, p. 267). Un claro ejemplo de esta situación que se explica en Naciones Unidas (2009) surge al analizar la prestación del servicio educativo: la administración del sistema educativo en un sentido general sí entraría dentro de la Letra $L$, mientras que la prestación de la enseñanza propiamente dicha corresponde a otro sector de actividad, por más que sea gestionada públicamente; lo mismo puede decirse de las actividades relacionadas al servicio de salud².

En otros términos, como detalla INDEC (2016), la administración pública comprende los tres niveles jurisdiccionales (nacional, provincial y municipal) y sus actividades se relacionan con la administración, justicia, defensa, seguridad, educación, salud, cultura y otros servicios de tipo social que no tienen un pago explícito o, si lo tienen, los precios generalmente no cubren el costo de producirlos (producción de no mercado). "Se incluyen los sistemas oficiales de previsión y los órganos de los poderes Legislativo y Judicial, así como algunas unidades productivas (no constituidas en sociedad) que producen bienes y servicios para el propio gobierno y/o para su venta en pequeña escala." (INDEC, 2016, p.58).

De acuerdo a INDEC (2016), los servicios que se producen en este sector pueden ser de dos tipos: de consumo colectivo, o de consumo individual. Los primeros tienen como fin beneficiar al conjunto de la sociedad (defensa, ordenación del territorio) y resulta imposible estimar el consumo que cada hogar hace individualmente de ellos. Los segundos son utilizados por personas y hogares (servicios sociales, salud, educación) y sí puede estimarse el uso que hace de ellos cada hogar.

\section{Revisión Metodológica}

\section{Metodología de cálculo de la Letra L del Producto Bruto Geográfico del Chaco}

El cálculo de la letra L, Administración pública, defensa y planes de seguridad social obligatoria, se basa en la metodología de las estimaciones del PBG 1993/2008 base 1993

\footnotetext{
${ }^{2}$ Vale mencionar que en la CIIU Revisión 4 el sector Administración pública y defensa y planes de seguridad social de afiliación obligatoria constituye la Letra O, en lugar de la Letra L como es mencionado en la CIIU 3.1. La versión 3.1 de la CIIU sirvió como base al clasificador de actividades utilizado para la realización del Censo Nacional Económico 2004, el cual constituye la principal fuente de información de las estadísticas provinciales y nacionales base 2004 para la mayoría de los sectores, por lo que para la revisión metodológica realizada aquí se hace referencia a tal clasificación.

"Visión de Futuro" Año 18, Volumen No 25 № 2, Edición Especial-III Congreso Regional de Economía del Norte Grande-Pág 80-100 URL de la Revista: http://visiondefuturo.fce.unam.edu.ar/index.php/visiondefuturo/index

URL del Documento: https://visiondefuturo.fce.unam.edu.ar/index.php/visiondefuturo/issue/view/20

ISSN 1668 - 8708 - Versión en Línea

E-mail: revistacientifica@fce.unam.edu.ar
} 
planteadas en el Informe Final de la CEPAL Anexo I (2009), hasta el último año calculado, 2016.

"Como la administración pública es un producto de no mercado, su producción se valúa por los costos." (INDEC, 2016, p.58). Así, la metodología utilizada propone, para obtener el Valor Bruto de Producción (VBP), estimar primero el Valor Agregado Bruto (VAB) y el Consumo Intermedio $(\mathrm{Cl})$, resultando el Valor Bruto de Producción como la suma de estos dos últimos. ${ }^{3}$

En término generales, la principal fuente de información, para las estimaciones a valores corrientes, la constituyen las ejecuciones presupuestarias de los diferentes niveles de gobierno, donde se observa principalmente el gasto en personal (que es el representativo del VAB) y el gasto en bienes y servicios no personales, que constituye el consumo intermedio. Para las estimaciones a valores constantes se recurre tanto a índices de volumen físico que permiten extrapolar o índices de precios para deflactar valores.

A continuación se enumeran los principales aspectos de la metodología actual, explicando lo que proponía la metodología de CEPAL (2009) y señalando lo que se hace actualmente, explicando las causas que llevaron a los cambios.

Año Base. Actualmente, el año base de la serie del PBG de la Provincia del Chaco es el año 2004. Anteriormente, el año base era 1993. La actualización de la base del cálculo provincial se realizó en 2013, cuando aún no estaba publicado el cambio de base realizado por el INDEC PIB. Por tal motivo, no se encontraba disponible en ese momento la metodología que seguiría este organismo para cambiar la base del PIB.

Los valores para el año base 2004 se obtuvieron de las estimaciones a precios corrientes realizadas para el año 2004 en el cálculo anterior (base 1993). Como se detalla en CEPAL (2009), las estimaciones a precios corrientes se apoyan en los estados de ejecución presupuestaria del Gobierno Provincial y Gobiernos Municipales, y en la asignación geográfica de los gastos del Gobierno Nacional que hasta el año 2006 fue realizada periódicamente por la Dirección Nacional de Cuentas Nacionales; y por último para el caso de la rama de Obras Sociales las estimaciones a valores corrientes se apoyaban en datos de la Encuesta Nacional de Grandes Empresas del INDEC.

\footnotetext{
${ }^{3}$ Esto se resalta ya que para otros sectores se calcula primero el Valor Bruto de Producción y el Consumo Intermedio y luego de se obtiene el Valor Agregado Bruto como resultado de la resta del primero con el segundo. $\mathrm{VAB}=\mathrm{VBP}-\mathrm{Cl}$; ó $\mathrm{VBP}=\mathrm{Cl}+\mathrm{VAB}$.

"Visión de Futuro" Año 18, Volumen No 25 N² 2, Edición Especial-III Congreso Regional de Economía del Norte Grande-Pág 80-100 URL de la Revista: http://visiondefuturo.fce.unam.edu.ar/index.php/visiondefuturo/index

URL del Documento: https://visiondefuturo.fce.unam.edu.ar/index.php/visiondefuturo/issue/view/20

ISSN 1668 - 8708 - Versión en Línea

E-mail: revistacientifica@fce.unam.edu.ar
} 


\section{Estimaciones a precios constantes}

En cuanto a las estimaciones a valores constantes, lo propuesto por CEPAL para el cálculo base 1993 consistía en la extrapolación de los valores correspondientes al año base mediante índices de volumen físico, o en deflactar los valores corrientes con índices de precios representativos:

- En lo que respecta a las estimaciones de valor agregado y consumo intermedio de la rama correspondiente a Gobierno Nacional se utilizaba el Índice de Precios Implícitos (IPI) del país, teniendo como fuente la Dirección Nacional de Cuentas Nacionales.

- En cuanto a la rama correspondiente a Gobierno Provincial, para extrapolar el valor agregado se utilizaba un índice de volumen físico (IVF) construido a partir de la planta ocupada. Por otro lado, para hallar el consumo intermedio a valores constantes se deflactaba el consumo intermedio con el Índice de Precios al Consumidor (IPC) de Resistencia, en su nivel general.

- Pasando al cálculo de la rama correspondiente al Gobierno Municipal también el valor agregado a precios constantes se estima extrapolando el valor del año base mediante un índice de volumen físico construido con la planta ocupada, y para el consumo intermedio se utiliza el IPC Resistencia para deflactar los valores.

- Por último, para la rama correspondiente a Obras Sociales se utilizó el IPI o el IVF correspondiente a la Letra L para el país.

Como se detalla en la sección siguiente, actualmente el cálculo se apartó de esta metodología en todas las ramas salvo en la correspondiente al Gobierno Provincial, dado que a partir del año 2008 se perdió continuidad en la información nacional y municipal que permitía el cálculo a valores corrientes. Así, en el momento el cálculo se realiza estimando la rama de Gobierno Provincial a valores constantes y luego calculando las demás de acuerdo a los porcentajes promedio de participación en el total.

Los datos que permiten construir el IVF a partir de la planta ocupada provincial se obtienen de la página web del Ministerio de Hacienda y Finanzas Públicas, donde se publica la planta ocupada al 31 de diciembre de cada año. Del número total de trabajadores que se detallan se apartan los correspondientes al Ministerio de Educación ${ }^{4}$ y al Ministerio de Salud,

\footnotetext{
${ }^{4}$ Para el caso del Ministerio de Educación la información permite distinguir entre los empleados con cargos en el escalafón docente y aquellos con cargos del escalafón general. Los primeros no son considerados para el cálculo de la Letra $L$, ya que corresponden a la actividad de enseñanza "Visión de Futuro" Año 18, Volumen $N^{\circ} 25$ № 2, Edición Especial-III Congreso Regional de Economía del Norte Grande-Pág 80-100 URL de la Revista: http://visiondefuturo.fce.unam.edu.ar/index.php/visiondefuturo/index

URL del Documento: https://visiondefuturo.fce.unam.edu.ar/index.php/visiondefuturo/issue/view/20

ISSN 1668 - 8708 - Versión en Línea

E-mail: revistacientifica@fce.unam.edu.ar
} 
ya que corresponden a otras actividades diferentes de las consideradas en la Letra L, como se explicó en la sección correspondiente a Descripción del Sector.

\section{Estimaciones a precios corrientes}

De acuerdo a la metodología original, "los cálculos a precios corrientes se apoyan en los estados de ejecución presupuestaria del Gobierno Provincial y Gobiernos Municipales, y en la asignación geográfica de los gastos del Gobierno Nacional que hasta el año 2006 fue realizada periódicamente por la DNCN" (CEPAL, 2009). En lo que concierne a Obras Sociales las estimaciones a precios corrientes eran calculadas en base a la Encuesta Nacional de Grandes Empresas (ENGE) del INDEC.

Desde el año 2008, la única fuente de información con la que se cuenta de manera continua la constituyen los estados de ejecución presupuestaria del Gobierno Provincial, que también son publicados en el sitio web del Ministerio de Hacienda y Finanzas Públicas. De estos estados, se toma el Gasto en Personal (los gastos en remuneraciones correspondientes a las funciones de educación y salud no son considerados por los motivos ya expresados, salvo los que se refieren a la administración de la salud y de la educación) como representativo del VAB a precios corrientes correspondiente a la actividad.

Una vez calculado el valor corriente del Gobierno Provincial, el resto de las ramas se estima mediante porcentajes promedio de participación en el total.

El gasto en bienes de consumo y servicios no personales también se obtiene de los estados de ejecución del gobierno provincial, y representa el consumo intermedio.

\section{Valuación}

Siguiendo la metodología detallada anteriormente, se obtiene el valor agregado bruto (VAB) a precios productor.

\section{Economía no observada}

Para el caso particular del cálculo de esta letra, al tratarse de actividades públicas gestionadas por los diferentes niveles de gobierno, no existiría el problema de la economía no observada que puede llevar a subestimaciones, como sí sucede en otras ramas.

propiamente dicha; mientras que los segundos sí son considerados para la estimación de la Letra L, ya que corresponden a la administración del sistema educativo.

"Visión de Futuro" Año 18, Volumen No 25 N 2, Edición Especial-III Congreso Regional de Economía del Norte Grande-Pág 80-100 URL de la Revista: http://visiondefuturo.fce.unam.edu.ar/index.php/visiondefuturo/index

URL del Documento: https://visiondefuturo.fce.unam.edu.ar/index.php/visiondefuturo/issue/view/20

ISSN 1668 - 8708 - Versión en Línea

E-mail: revistacientifica@fce.unam.edu.ar 


\section{Metodología de cálculo de la Letra $L$ del Producto Interno Bruto de Argentina}

Se resumen a continuación las principales características de la metodología utilizada por Nación para el cálculo de la Letra L base 2004, descrita en INDEC (2016).

\section{Año Base}

Igual que el PBG, el actual año base del PIB es el año 2004 y anteriormente era el año 1993. El cambio del año base se realizó siguiendo los conceptos y recomendaciones del Sistema de Cuentas Nacionales 2008. (INDEC, 2016).

La metodología detalla las diferentes fuentes de información a las que se recurrió para la estimación de la Administración Pública Nacional, Provincial y Municipal.

Para la primera, el cálculo se apoya principalmente en la ejecución presupuestaria del Sistema Integrado de Información Financiera (SIDIF) y en la Cuenta de Inversión de la Nación. De manera similar a lo detallado en el apartado correspondiente al PBG el cálculo del VAB está compuesto por el gasto en remuneraciones, y el $\mathrm{Cl}$ se refiere al gasto en bienes de consumo y servicios no personales. Luego de la suma del VAB y el Cl se obtiene el VBP.

En cuanto a la Administración Pública Provincial, "el objetivo de máxima fue replicar la metodología aplicada para la APN en todas aquellas provincias que contaran con información según los requisitos de desagregación y detalle conceptual utilizados a nivel nacional" (INDEC, 2016, p.60). Vale destacar que la provincia del Chaco no es mencionada entre estas provincias, por lo que se ocupó el criterio de finalidad-función ajustado (INDEC, 2016).

En la metodología se explica que la clasificación presupuestaria del gasto por finalidad-función tiene una limitación: no es posible distinguir entre prestación de servicios colectivos o individuales (como las actividades de enseñanza, por ejemplo). Para solucionar este problema, se utilizó información muestral adicional, para estimar qué proporción de los gastos agrupados en una finalidad-función correspondían a la burocracia administrativa y qué parte a la prestación efectiva de un servicio individual (INDEC, 2016). La explicación de estos ajustes es relevante porque para el caso de estimación del PBG, la ejecución del gasto provincial es publicada justamente según finalidad y función.

"Para la estimación de la APM se utilizaron los datos de ejecución presupuestaria del año 2004. Para determinar la estructura de gastos por actividad se recurrió a información recopilada para la MIP 97 por la DNCN" (INDEC, 2016, p. 60).

En cuanto al consumo intermedio, la estimación para el año base se basó en ejecuciones presupuestarias por actividad y por nivel jurisdiccional. También se elaboró un vector para el Cl según clasificación de productos. (INDEC, 2016).

\footnotetext{
"Visión de Futuro" Año 18, Volumen $\mathbf{N}^{\circ} 25$ № 2, Edición Especial-III Congreso Regional de Economía del Norte Grande-Pág 80-100 URL de la Revista: http://visiondefuturo.fce.unam.edu.ar/index.php/visiondefuturo/index

URL del Documento: https://visiondefuturo.fce.unam.edu.ar/index.php/visiondefuturo/issue/view/20

ISSN 1668 - 8708 - Versión en Línea

E-mail: revistacientifica@fce.unam.edu.ar
} 


\section{Estimaciones a precios constantes}

El VAB se obtiene extrapolando el valor del año base por un IVF basado en la cantidad de trabajadores de la administración pública, para cada nivel de gobierno. Para el nivel nacional, las fuentes consultadas son el Sistema Integrado Previsional Argentina (SIPA) y Dirección Nacional de Ocupación y Salarios del Sector Público (DNOySSP). Para la administración provincial las fuentes consultadas para construir el índice son: la Dirección Nacional de Coordinación Fiscal con las Provincias, el SIPA, o estimaciones en base a información provista por cada provincia. Para la administración municipal, la fuente es el SIPA.

"Para estimar el $\mathrm{Cl}$ a precios constantes, se deflacta el $\mathrm{Cl}$ a precios corrientes por actividad económica y por nivel jurisdiccional con un índice de precios propio del sector elaborado en la DNCN" (INDEC, 2016, p.61).

\section{Estimaciones a precios corrientes}

Para la administración pública nacional, de igual manera que para el cálculo de los valores del año base, el VAB y el Cl se obtienen de datos del SIDIF y de la Cuenta Inversión.

Para el caso de las provincias, la estimación del VAB y el $\mathrm{Cl}$, depende de la calidad de información con la que se cuente a la fecha de cierre. Según esto, o se estima siguiendo la misma metodología que para el año base, o se recurre a índices de valor construidos por la Dirección Nacional de Cuentas Nacionales.

"El valor del VAB de la Administración Pública Municipal (APM) se obtiene a través de información publicada por la DNCFP y en los períodos donde no se cuenta con dicha información, se utiliza un IV que refleja la evolución del costo salarial del SIPA tanto anual como trimestral. Las estimaciones del $\mathrm{Cl}$ se basan en datos de la DNCFP y para los trimestres donde la información no está disponible, se calcula suponiendo coeficiente fijo respecto del VBP" (INDEC, 2016, p.62).

\section{Valuación}

Siguiendo la metodología detallada anteriormente, se obtiene el VAB a precios básicos.

\section{Economía no observada}

En el apartado correspondiente a la estimación de la letra L, INDEC (2016) no se mencionan aspectos relacionados a la economía no observada. Como se explicó en el apartado correspondiente al PBG, al tratarse de actividades llevadas a cabo por el sector público, no deberían presentarse problemas respecto a la economía no observada, como sí se presentan en otras ramas, donde sí se puede dar lugar a producción oculta, producción ilegal o informal.

\footnotetext{
"Visión de Futuro" Año 18, Volumen No 25 № 2, Edición Especial-III Congreso Regional de Economía del Norte Grande-Pág 80-100 URL de la Revista: http://visiondefuturo.fce.unam.edu.ar/index.php/visiondefuturo/index

URL del Documento: https://visiondefuturo.fce.unam.edu.ar/index.php/visiondefuturo/issue/view/20 


\section{Revisión del nomenclador}

Otro aspecto relevante a la hora de analizar la metodología de estimación de las ramas de actividad es la actualización de los nomencladores.

Los nomencladores utilizados en el SCN son la Clasificación Industrial Internacional Uniforme de todas las actividades económicas (CIIU) y la Clasificación Central de Productos (CCP). Su rol en las estimaciones no es menor porque "los clasificadores o nomenclaturas permiten la comparabilidad internacional e intertemporal en el ámbito nacional de la estadística económica" (INDEC, 2016, p.28).

En lo que concierne a las actividades, en Argentina, para el cálculo del PIB base 2004, como ya se mencionó, la CIIU revisión 3.1 es la base de la Clasificación Nacional de Actividades Económicas 2004 (ClaNAE 04) utilizada por el INDEC en el último Censo Nacional Económico 2004/2005 (INDEC, 2016).

Mientras que para el cálculo del PBG vigente el nomenclador utilizado ha sido CLaNAE 97. Por otra parte, el INDEC puso en vigencia en el año 2010 el CLaNAE 2010 basado en la CIIU revisión 4.

Pasando al análisis de la actividad "Administración pública y defensa, y planes de seguridad social de afiliación obligatoria", cuando se realiza una comparación general del CLaNAE 97 (utilizado actualmente para el cálculo del PBG) y el 2010, (que sería el vigente actualmente y el que contiene todas las actualizaciones propuestas por el CIIU revisión 4), podemos percibir un cambio en el orden de clasificación de las actividades de "servicios de justicia" y "servicio para el orden público y la seguridad". Como así también vemos que esta actividad en su última versión se encuentra clasificada dentro de una nueva letra, la letra $\mathrm{O}$, y con un orden distinto a la versión utilizada actualmente.

Ahora bien, cuando realizamos una inspección más detallada podemos notar por ejemplo diferencias en las siguientes actividades entre la CLaNAE 97 y 2010 :

- 84.120 Servicios para la regulación de las actividades sanitarias, educativas, culturales, y restantes servicios sociales, excepto seguridad social obligatoria: en el nuevo nomenclador incluye "El patrocinio de actividades recreativas y culturales, la concesión de becas a artistas, la administración de programas y servicios para: el suministro de agua potable, recolección y eliminación de residuos, protección ambiental y vivienda" (INDEC, 2011, p.397).

- 84.13 Servicios para la regulación de la actividad económica: podemos notar la incorporación "las actividades de la Comisión Nacional de valores CNV. Por tratarse de un ente público de control” (INDEC, 2011, p. 398)

\footnotetext{
"Visión de Futuro" Año 18, Volumen N$^{\circ} 25$ № 2, Edición Especial-III Congreso Regional de Economía del Norte Grande-Pág 80-100 URL de la Revista: http://visiondefuturo.fce.unam.edu.ar/index.php/visiondefuturo/index

URL del Documento: https://visiondefuturo.fce.unam.edu.ar/index.php/visiondefuturo/issue/view/20

ISSN 1668 - 8708 - Versión en Línea

E-mail: revistacientifica@fce.unam.edu.ar
} 
- $\quad 84.190$ Servicios auxiliares para los servicios generales de la Administración Pública n.c.p.: podemos notar que en la actualización se excluyen "Las actividades de archivos cinematográficos".

- 84.210 Servicios de Asuntos exteriores: en esta sección la última revisión incluye una nueva actividad para el cálculo el suministro de ayuda militar a otros países pero también excluye los servicios de socorro internacional a refugiados como consecuencia de desastres o conflictos.

- 84.230 Servicios para el orden público y la seguridad: a diferencia del nomenclador utilizado actualmente por la provincia, en la actualización se excluyen las actividades de laboratorios policiales.

- 84.3 Servicios de la seguridad social obligatoria, excepto obras sociales: las modificaciones en esta actividad son varias y la mayoría responden a la excepción de las obras sociales. También presenta en la actualización una definición más clara y concreta de que se debe incluir.

Como se puede notar, haciendo un análisis hasta tres dígitos las discrepancias entre el nomenclador utilizado actualmente y la última actualización no son significativas, en razón que no se presenta un nuevo grupo para calcular o se elimina alguno, lo que puede observase en los siguientes cuadros. Pero, cuando se realiza una inspección más detallada dentro de cada subdivisión y grupo podemos notar múltiples modificaciones respecto de qué se incluye o no para el cálculo de cada una de ellas.

Tabla 1. Actividades de la Letra O en el CIIU Revisión 4.

\begin{tabular}{|c|c|c|c|}
\hline $\begin{array}{c}\text { O. ADMINISTRACION PUBLICA, } \\
\text { DEFENSA Y SEGURIDAD SOCIAL } \\
\text { OBLIGATORIA }\end{array}$ & CIIU REV 4 & & \\
\hline Sección & & & $\begin{array}{c}\text { Descripción } \\
\text { Administración pública y } \\
\text { defensa; planes de seguridad } \\
\text { social } \\
\text { de afiliación obligatoria }\end{array}$ \\
\hline 84 & División Grupo & Clase & $\begin{array}{c}\text { Administración del Estado y } \\
\text { aplicación de la política } \\
\text { económica y social de la } \\
\text { comunidad }\end{array}$ \\
\hline
\end{tabular}

\footnotetext{
"Visión de Futuro" Año 18, Volumen $\mathrm{N}^{0} 25$ N 2, Edición Especial-III Congreso Regional de Economía del Norte Grande-Pág 80-100 URL de la Revista: http://visiondefuturo.fce.unam.edu.ar/index.php/visiondefuturo/index

URL del Documento: https://visiondefuturo.fce.unam.edu.ar/index.php/visiondefuturo/issue/view/20

ISSN 1668 - 8708 - Versión en Línea

E-mail: revistacientifica@fce.unam.edu.ar
} 


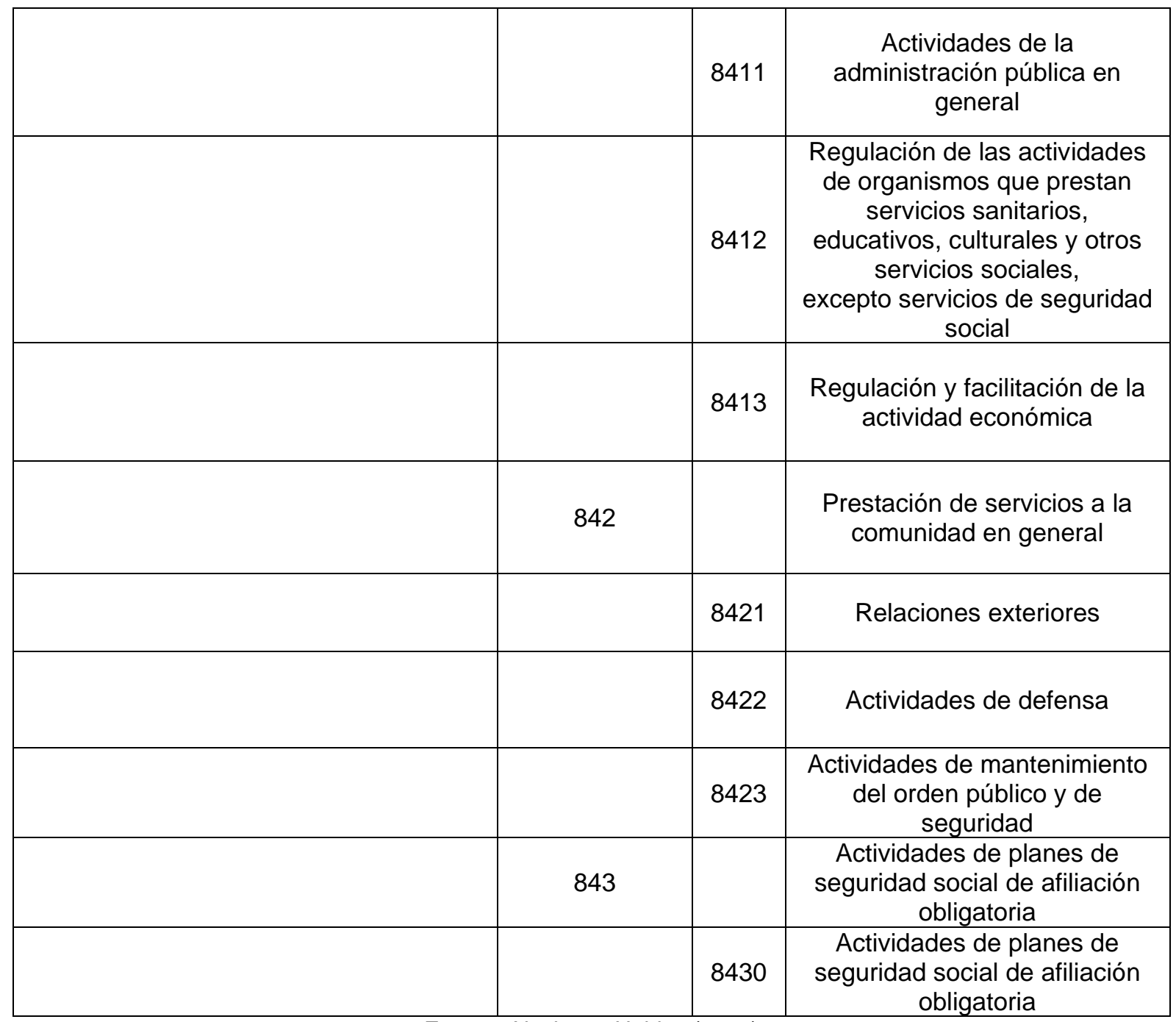

Fuente: Naciones Unidas (2008)

Tabla 2. Letra L en la CLaNAE 97

\begin{tabular}{|c|c|c|c|}
\hline $\begin{array}{c}\text { L ADMINISTRACIÓN PÚBLICA, } \\
\text { DEFENSA Y SEGURIDAD SOCIAL } \\
\text { OBLIGATORIA }\end{array}$ & \multicolumn{2}{|c|}{ CLANAE 97 } & \\
\hline Sección & División Grupo & Clase & Descripción \\
\hline 75 & 751 & & $\begin{array}{c}\text { ADMINISTRACIÓN PÚBLICA, } \\
\text { DEFENSA Y SEGURIDAD } \\
\text { SOCIAL OBLIGATORIA }\end{array}$ \\
\hline & & & $\begin{array}{c}\text { Servicios de la Administración } \\
\text { Pública }\end{array}$ \\
\hline
\end{tabular}

\footnotetext{
"Visión de Futuro" Año 18, Volumen $\mathrm{N}^{\circ} 25$ N 2, Edición Especial-III Congreso Regional de Economía del Norte Grande-Pág 80-100 URL de la Revista: http://visiondefuturo.fce.unam.edu.ar/index.php/visiondefuturo/index

URL del Documento: https://visiondefuturo.fce.unam.edu.ar/index.php/visiondefuturo/issue/view/20

ISSN 1668 - 8708 - Versión en Línea

E-mail: revistacientifica@fce.unam.edu.ar
} 


\begin{tabular}{|c|c|c|c|}
\hline & & 7511 & $\begin{array}{l}\text { Servicios generales de la } \\
\text { Administración Pública }\end{array}$ \\
\hline & & 7512 & $\begin{array}{c}\text { Servicios para la regulación de las } \\
\text { actividades sanitarias, educativas, } \\
\text { culturales, y } \\
\text { restantes servicios sociales, } \\
\text { excepto seguridad social } \\
\text { obligatoria }\end{array}$ \\
\hline & & 7513 & $\begin{array}{c}\text { Servicios para la regulación de la } \\
\text { actividad económica }\end{array}$ \\
\hline & & 751.9 & $\begin{array}{c}\text { Servicios auxiliares para los } \\
\text { servicios generales de la } \\
\text { Administración Pública } \\
\text { n.c.p. }\end{array}$ \\
\hline & 752 & & $\begin{array}{c}\text { Prestación Pública de servicios a la } \\
\text { comunidad en general }\end{array}$ \\
\hline & & 7521 & Servicios de Asuntos exteriores \\
\hline & & 7522 & Servicios de defensa \\
\hline & & 7523 & Servicios de justicia \\
\hline & & 7524 & $\begin{array}{l}\text { Servicios para el orden público y la } \\
\text { seguridad }\end{array}$ \\
\hline & & 7525 & Servicios de protección civil \\
\hline & 753 & & $\begin{array}{l}\text { Servicios de la seguridad social } \\
\text { obligatoria }\end{array}$ \\
\hline & & 7530 & $\begin{array}{c}\text { Servicios de la seguridad social } \\
\text { obligatoria }\end{array}$ \\
\hline
\end{tabular}

Fuente: INDEC (2011)

Tabla 3. Letra O en la ClaNAE 2010

\begin{tabular}{|c|c|c|c|}
\hline $\begin{array}{c}\text { O. ADMINISTRACIÓN PÚBLICA, } \\
\text { DEFENSA Y SEGURIDAD SOCIAL } \\
\text { OBLIGATORIA }\end{array}$ & \multicolumn{2}{|c|}{ ClaNAE 2010 } \\
\hline Sección & División Grupo & Clase & \\
\hline 84 & & & $\begin{array}{c}\text { Descripción } \\
\text { ADMINISTRACIÓN PÚBLICA, } \\
\text { DEFENSA Y SEGURIDAD } \\
\text { SOCIAL OBLIGATORIA }\end{array}$ \\
\hline
\end{tabular}

"Visión de Futuro" Año 18, Volumen No 25 № 2, Edición Especial-III Congreso Regional de Economía del Norte Grande-Pág 80-100 URL de la Revista: http://visiondefuturo.fce.unam.edu.ar/index.php/visiondefuturo/index

URL del Documento: https://visiondefuturo.fce.unam.edu.ar/index.php/visiondefuturo/issue/view/20

ISSN 1668 - 8708 - Versión en Línea

E-mail: revistacientifica@fce.unam.edu.ar 


\begin{tabular}{|c|c|c|c|}
\hline & 841 & & $\begin{array}{c}\text { Servicios de la Administración } \\
\text { Pública }\end{array}$ \\
\hline & & 8411 & $\begin{array}{l}\text { Servicios generales de la } \\
\text { Administración Pública }\end{array}$ \\
\hline & & 8412 & $\begin{array}{c}\text { Servicios para la regulación de las } \\
\text { actividades sanitarias, educativas, } \\
\text { culturales, y } \\
\text { restantes servicios sociales, } \\
\text { excepto seguridad social } \\
\text { obligatoria }\end{array}$ \\
\hline & & 8413 & $\begin{array}{c}\text { Servicios para la regulación de la } \\
\text { actividad económica }\end{array}$ \\
\hline & & 841.9 & $\begin{array}{l}\text { Servicios auxiliares para los } \\
\text { servicios generales de la } \\
\text { Administración Pública } \\
\text { n.c.p. }\end{array}$ \\
\hline & 842 & & $\begin{array}{l}\text { Prestación Pública de servicios a la } \\
\text { comunidad en general }\end{array}$ \\
\hline & & 8421 & Servicios de Asuntos exteriores \\
\hline & & 8422 & Servicios de defensa \\
\hline & & 8423 & $\begin{array}{l}\text { Servicios para el orden público y la } \\
\text { seguridad }\end{array}$ \\
\hline & & 8424 & Servicios de justicia \\
\hline & & 8425 & Servicios de protección civil \\
\hline & 843 & & $\begin{array}{l}\text { Servicios de la seguridad social } \\
\text { obligatoria, excepto obras sociales }\end{array}$ \\
\hline & & 8430 & $\begin{array}{c}\text { Servicios de la seguridad social } \\
\text { obligatoria }\end{array}$ \\
\hline
\end{tabular}

Fuente: INDEC (2011)

\section{Análisis de las estimaciones}

A continuación se expone la serie de datos correspondiente al valor agregado de la Letra L para el Chaco, y para Argentina, calculado de acuerdo a las metodologías detalladas anteriormente, a precios del año base 2004.

\footnotetext{
"Visión de Futuro" Año 18, Volumen $\mathrm{N}^{\circ} 25$ N 2, Edición Especial-III Congreso Regional de Economía del Norte Grande-Pág 80-100 URL de la Revista: http://visiondefuturo.fce.unam.edu.ar/index.php/visiondefuturo/index

URL del Documento: https://visiondefuturo.fce.unam.edu.ar/index.php/visiondefuturo/issue/view/20

ISSN 1668 - 8708 - Versión en Línea

E-mail: revistacientifica@fce.unam.edu.ar
} 
Tabla 4. Argentina. Letra L. Valor Agregado Bruto a precios básicos. Año base 2004. En millones de pesos.

\begin{tabular}{|r|r|c|}
\hline Año & Letra L & Variación \\
\hline 2004 & 21.585 & \\
\hline 2005 & 22.732 & $5 \%$ \\
\hline 2006 & 23.503 & $3 \%$ \\
\hline 2007 & 24.825 & $6 \%$ \\
\hline 2008 & 25.848 & $4 \%$ \\
\hline 2009 & 26.689 & $3 \%$ \\
\hline 2010 & 27.611 & $3 \%$ \\
\hline 2011 & 28.489 & $3 \%$ \\
\hline 2012 & 29.346 & $3 \%$ \\
\hline 2013 & 30.121 & $3 \%$ \\
\hline 2014 & 31.055 & $3 \%$ \\
\hline 2015 & 32.220 & $4 \%$ \\
\hline
\end{tabular}

Fuente: Elaboración propia en base a datos del INDEC

Tabla 5. Chaco. Letra L. Valor Agregado Bruto a precios productor. Año base 2004. En miles de pesos.

\begin{tabular}{|r|r|c|}
\hline Año & Letra $\mathrm{L}$ & Variación \\
\hline 2004 & 501.525 & \\
\hline 2005 & 507.990 & $1 \%$ \\
\hline 2006 & 510.376 & $0 \%$ \\
\hline 2007 & 542.318 & $6 \%$ \\
\hline 2008 & 591.022 & $9 \%$ \\
\hline 2009 & 625.250 & $6 \%$ \\
\hline 2010 & 670.118 & $7 \%$ \\
\hline 2011 & 695.187 & $4 \%$ \\
\hline 2012 & 731.851 & $5 \%$ \\
\hline 2013 & 754.898 & $3 \%$ \\
\hline 2014 & 774.252 & $3 \%$ \\
\hline 2015 & 800.122 & $3 \%$ \\
\hline
\end{tabular}

Fuente: Elaboración propia en base a datos de la Dirección de Estadística y Censo.

Si bien estas series no son directamente comparables, dado que el comportamiento de las actividades de la Letra $L$ en la provincia del Chaco no es necesariamente igual a lo que sucede si se tiene en cuenta a la Argentina en su conjunto, y también la participación del Chaco en el total es baja, resulta interesante observar las variaciones anuales del valor agregado en cada caso. Como puede observarse en algunos años las tasas de crecimiento resultan muy diferentes.

Sería conveniente revisar esos años, de manera de concluir si esas diferencias se deben a cuestiones coyunturales o estructurales propias de las actividades de la provincia, que no

\footnotetext{
"Visión de Futuro" Año 18, Volumen No 25 № 2, Edición Especial-III Congreso Regional de Economía del Norte Grande-Pág 80-100 URL de la Revista: http://visiondefuturo.fce.unam.edu.ar/index.php/visiondefuturo/index

URL del Documento: https://visiondefuturo.fce.unam.edu.ar/index.php/visiondefuturo/issue/view/20

ISSN 1668 - 8708 - Versión en Línea

E-mail: revistacientifica@fce.unam.edu.ar
} 
se condicen con lo que sucedía a nivel nacional, o si se trata de errores en las estimaciones, posiblemente por falta de información.

Otra cuestión que surge al analizar la serie provincial, es que la participación de la Letra L en el PBG del Chaco fue disminuyendo. En 2004, el año base, la participación de la Letra $L$ era del 9,71\% mientras que en el año 2015 cae a 6,81\%. Esta caída debe ser analizada junto con el comportamiento de las otras letras. Es llamativa porque la provincia del Chaco cuenta con un sector público amplio, donde trabajan aproximadamente 37.000 personas, sin contar los docentes.

\section{CONCLUSIÓN}

Esta primera revisión de las metodologías de cálculo de la letra L permite establecer una serie de consideraciones finales. Se trata de las principales cuestiones que deben ser revisadas en las estimaciones del PBG de la Provincia del Chaco, específicamente en lo que concierne al sector público. A su vez, esta revisión metodológica plantea cuáles podrían ser los pasos a seguir en cuanto al mejoramiento del cálculo de este indicador y en la posterior generación de una nueva metodología actualizada.

En cuanto al cambio del año base, la estimación de la Letra $L$ en el PBG surgió de las estimaciones a valores corrientes para el año 2004 que se habían calculado para el PBG base 1993. A diferencia de otras letras, donde la fuente principal de información para la nueva base fue el Censo Nacional Económico 2004, y donde la provincia no contó con información para ajustar los valores del censo (que correspondían a 2003) al 2004, ni datos para considerar la participación de la economía no observada; la Letra $L$ tienen como fuente principal de información los estados de ejecución presupuestaria de los distintos niveles de gobierno, por lo que la estimación de los valores corrientes2004 hecha para el PBG base 1993 es válida como estimación de los valores para el nuevo año base.

Sin embargo, cuando se analizan los datos que posibilitaron el cálculo, se observan carencias de información para el año 2004 especialmente en lo que se refiere a administración pública municipal. Para la mayoría de los municipios no se contaba con los datos de gasto en personal y en bienes de consumo y servicios no personales, y estos datos fueron estimados en base al comportamiento de estos valores en ejecuciones presupuestarias de otros años. Por lo tanto, las estimaciones de $\mathrm{VAB}$ y $\mathrm{Cl}$ de la base podrían reestimarse si se consigue nueva información.

Continuando con el análisis metodológico, no parecería haber grandes discrepancias con lo utilizado por Nación. Al tratarse de una producción que no está dirigida al mercado, el valor bruto de producción se obtiene como suma del valor agregado bruto y del consumo

\footnotetext{
"Visión de Futuro" Año 18, Volumen N 25 N² 2, Edición Especial-III Congreso Regional de Economía del Norte Grande-Pág 80-100 URL de la Revista: http://visiondefuturo.fce.unam.edu.ar/index.php/visiondefuturo/index

URL del Documento: https://visiondefuturo.fce.unam.edu.ar/index.php/visiondefuturo/issue/view/20

ISSN 1668 - 8708 - Versión en Línea

E-mail: revistacientifica@fce.unam.edu.ar
} 
intermedio. Tanto para el cálculo de la Letra $L$ en el PBG o en el PIB, y como ya se mencionó, la principal fuente de información para obtener ambos son los estados de ejecución de presupuestaria y, luego, los valores se extrapolan por índices de volumen físico realizados a partir de datos de personal o se deflactan por índices de precios representativos. Por lo tanto, en cuanto a la fuente para el año base y para los valores corrientes de cada año hay coincidencias en ambas metodologías, así como en la forma de realizar las estimaciones a valores constantes.

Teniendo en cuenta todo lo anterior, se considera que la investigación podría continuar por dos caminos. Una referente al acceso a información y otra referida a la mejora de la metodología. En primer lugar, se debe procurar conseguir información del gasto en personal y en bienes de consumo y servicios no personales de los diferentes municipios que integran la provincia, así como datos que permitan estimar qué parte del gasto de la administración nacional corresponde a la provincia del Chaco. También es preciso contar con datos para estimar la rama correspondiente a la seguridad social, dado que la provincia del Chaco cuenta con una caja previsional. Conseguir toda esta información resulta clave, tanto para revisar los valores correspondientes al año base, como para continuar con la estimación cada año, ya que en la actualidad los valores son estimados solamente de los datos de la administración provincial.

En segundo lugar, siguiendo la metodología de Nación, se podría clasificar por productos el gasto en bienes y servicios de la administración, de manera de utilizar así el índice de precios más representativo posible al momento de deflactar valores para obtener el consumo intermedio en términos constantes.

Finalmente, respecto al análisis de los nomencladores, la provincia trabaja con la CLaNAE 97, mientras que para el PBI se utiliza la CLaNAE 2004 y ya existe la CLaNAE 2010. Si bien esta letra no se vio fuertemente afectada por las modificaciones del nuevo nomenclador, no son menores las reformaciones propuestas ya que estas ayudan a mejorar el cálculo y sobre todo poder delimitar de manera más precisa que actividades conforman su cómputo.

Con el fin de poder lograr un ajuste y actualización metodológica para el cálculo de esta rama de actividad, se propone estudiar la viabilidad al momento de incorporar estas modificaciones debido a la falta de información que se puede presentar a la hora de su construcción, como también analizar cuáles de estas actividades incorporadas se realizan dentro del territorio provincial. Así también, se debería lograr una anexión progresiva de manera que el cómputo de la letra $L$ no se encuentre afectado de manera significativa por las modificaciones.

\footnotetext{
"Visión de Futuro" Año 18, Volumen No 25 N² 2, Edición Especial-III Congreso Regional de Economía del Norte Grande-Pág 80-100 URL de la Revista: http://visiondefuturo.fce.unam.edu.ar/index.php/visiondefuturo/index

URL del Documento: https://visiondefuturo.fce.unam.edu.ar/index.php/visiondefuturo/issue/view/20

ISSN 1668 - 8708 - Versión en Línea

E-mail: revistacientifica@fce.unam.edu.ar
} 


\section{REFERENCIAS BIBLIOGRÁFICAS}

CEPAL (2009). Informe Final. Anexo 1. Resultados y Metodología de las Estimaciones del Producto Geográfico Bruto 1993/2008. Convenio CEPAL/ Gobierno de la Provincia del Chaco.

Dirección Provincial de Estadística (2018). Producto Bruto Geográfico Provincia de Buenos Aires (PBG-PBA). Informe Metodológico Base 2004. Subsecretaría de Política y Coordinación Económica. Ministerio de Economía. Provincia de Buenos Aires. Recuperadode:http://www.estadistica.ec.gba.gov.ar/dpe/images/METODOLOGIA_PB G_PBA_BASE_2004.Pdf

INDEC (2011) Clasificador Nacional de Actividades Económicas CLANAE 1997 Notas Metodológicas. Recuperado de: https://www.indec.gob.ar/micro_sitios/clanae/documentos/NOTAS_METODOLOGICA S_CLANAE-1997.pdf

INDEC (2011) Clasificador Nacional de Actividades Económicas - CLANAE 2010 Notas Metodológicas. Recuperado de: https://www.indec.gob.ar/micro_sitios/clanae/documentos/NOTAS_METODOLOGICA S_CLANAE-2010.pdf

INDEC (2016). Cuentas Nacionales. Metodología de Estimación. Base 2004 y series a precios constantes y corrientes. Recuperado de: https://www.indec.gov.ar/ftp/cuadros/economia/metodologia_21_cuentas_nacionales. pdf

Laveglia, F.H. (2006) Contribuciones para la Formulación de un Sistema de Cuentas Regionales. Recuperado de: http://www.eumed.net/libros-gratis/2006c/211/index.htm Naciones Unidas. (2005).Clasificación Industrial Internacional Uniforme (CIIU) de todas las Actividades Económicas. (Informes Estadísticos - Serie M, n. 4, Rev. 3.1). Nueva York. Recuperado de: https://unstats.un.org/unsd/publication/seriesm/seriesm_4rev3_1s.pdf Naciones Unidas, OCDE, Comisión Europea y otros. (2016). Sistema de Cuentas Nacionales 2008. Nueva York. Recuperado de:https://unstats.un.org/unsd/nationalaccount/docs/sna2008spanish.pdf

Naciones Unidas. (2009).Clasificación Industrial Internacional Uniforme (CIIU) de todas las Actividades Económicas. (Informes Estadísticos - Serie M, n. 4, Rev. 4). Nueva York. Recuperado de: https://unstats.un.org/unsd/publication/seriesm/seriesm_4rev4s.pdf Séruzier, M (2003). Medir la economía de los países según el Sistema de Cuentas Nacionales.Naciones Unidas y CEPAL en coedición con Alfaomega colombiana S.A.

\footnotetext{
"Visión de Futuro" Año 18, Volumen Nº 25 N² 2, Edición Especial-III Congreso Regional de Economía del Norte Grande-Pág 80-100 URL de la Revista: http://visiondefuturo.fce.unam.edu.ar/index.php/visiondefuturo/index

URL del Documento: https://visiondefuturo.fce.unam.edu.ar/index.php/visiondefuturo/issue/view/20 
https://repositorio.cepal.org/bitstream/handle/11362/1800/1/S3393S489_es.pdf

\section{RESUMEN BIBLIOGRÁFICO}

\section{Alcira Patricia Soria Ojeda}

Esp. en Docencia Universitaria en Ciencias Empresariales y Sociales (UCES). Lic. en Economía (UNNE). Docente Titular en UCES sede Resistencia y Auxiliar Docente en la FCE de la UNNE. Docente Investigadora en la FCE de la UNNE en proyecto sobre estadísticas regionales y Producto Bruto Geográfico.

\section{Julieta Bermúdez}

Estudiante de Licenciatura en Economía, en la Facultad de Ciencias Económicas de la UNNE. Alumna anexa al proyecto de investigación "Hacia la construcción de estadísticas regionales actualizadas: revisión y asignación geográfica del producto bruto geográfico del chaco". Sus intereses de investigación incluyen estadísticas regionales y su metodología.

\footnotetext{
"Visión de Futuro" Año 18, Volumen № 25 № 2, Edición Especial-III Congreso Regional de Economía del Norte Grande-Pág 80-100 URL de la Revista: http://visiondefuturo.fce.unam.edu.ar/index.php/visiondefuturo/index

URL del Documento: https://visiondefuturo.fce.unam.edu.ar/index.php/visiondefuturo/issue/view/20

ISSN 1668 - 8708 - Versión en Línea

E-mail: revistacientifica@fce.unam.edu.ar
} 